ANALYSIS \& PDE Volume $7 \quad$ No. $3 \quad 2014$ (3) PIERRE JAMMES

PRESCRIPTION DU SPECTRE DE STEKLOV DANS UNE CLASSE CONFORME 


\title{
PRESCRIPTION DU SPECTRE DE STEKLOV DANS UNE CLASSE CONFORME
}

\author{
PIERRE JAMMES
}

\begin{abstract}
Sur toute variété compacte de dimension $n \geq 3$ à bord, on prescrit toute partie finie du spectre de Steklov dans une classe conforme donnée. En particulier, on prescrit la multiplicité des valeurs propres. Sur une surface compacte à bord donnée, on montre que la multiplicité de la $k$-ième valeur propre est bornée indépendamment de la métrique. Sur le disque, on donne des résultats plus précis : la multiplicité de la première et la deuxième valeurs propres non nulles sont au plus 2 et 3 respectivement. Pour le problème de Steklov-Neumann sur le disque, on montre que la multiplicité de la $k$-ième valeur propre non nulle est au plus $k+1$.

On any compact manifold of dimension $n \geq 3$ with boundary, we prescribe any finite part of the Steklov spectrum within a given conformal class. In particular, we prescribe the multiplicity of the first eigenvalues. On a compact surface with boundary, we show that the multiplicity of the $k$-th eigenvalue is bounded independently of the metric. On the disk, we give more precise results: the multiplicity of the first and second positive eigenvalues are at most 2 and 3 respectively. For the Steklov-Neumann problem on the disk, we prove that the multiplicity of the $k$-th positive eigenvalue is at most $k+1$.
\end{abstract}

\section{Introduction}

Étant donnée une variété riemannienne $(M, g)$ compacte à bord et une fonction strictement positive $\rho \in C^{0}(\partial M)$, le spectre de Steklov de $M$ est l'ensemble des réels $\sigma$ tels que le système

$$
\begin{cases}\Delta f=0 & \text { dans } M \\ \partial f / \partial v=\sigma \rho f & \text { sur } \partial M\end{cases}
$$

où $v$ est un vecteur normal unitaire sortant le long de $\partial M$, admette des solutions non triviales. Ce spectre est formé de valeurs propres positives notées $0=\sigma_{0}(M, g, \rho)<\sigma_{1}(M, g, \rho) \leq \sigma_{2}(M, g, \rho) \leq \cdots \rightarrow+\infty$. Si $\rho \equiv 1$, alors c'est aussi le spectre de l'opérateur Dirichlet-to-Neumann sur $M$.

Un grand nombre de travaux récents visent à borner ces valeurs propres sous des contraintes géométriques, par exemple avec des hypothèses conformes [Fraser et Schoen 2011; Hassannezhad 2011], ou en fonction d'une constante isopérimétrique [Colbois et al. 2011]. Le but de cet article vise au contraire à mettre en évidence le fait que ce spectre possède une certaine souplesse et que si la dimension de $M$ est au moins 3, on peut en prescrire toute partie finie, c'est-à-dire que si on se donne une suite finie de réels strictement positifs, il existe une métrique sur $M$ telle que cette suite soit le début du spectre. On va en fait montrer un résultat plus fort, à savoir qu'on peut prescrire simultanément le début du spectre, la multiplicité des valeurs propres, la classe conforme de la variété et la fonction densité $\rho$ sur le bord.

MSC2010: 35P15, 58J50.

Mots-clefs : Steklov eigenvalues, prescription of eigenvalues, conformal geometry. 
On étend ainsi au spectre de Steklov un résultat obtenu dans [1987] pour le laplacien et généralisé aux formes différentielles dans [Jammes 2011].

Théorème 1.2. Soit $\left(M^{n}, g\right)$ une variété riemannienne compacte à bord de dimension $n \geq 3$. Étant donnée une fonction strictement positive $\rho \in C^{0}(\partial M)$, un entier $N \geq 1$ et une suite finie de réels strictement positifs $0<a_{1} \leq a_{2} \leq \cdots \leq a_{N}$, il existe une métrique $\tilde{g}$ conforme à g telle que

$$
\sigma_{k}(M, \rho, \tilde{g})=a_{k}
$$

pour tout $k \in[1, N]$.

Remarque 1.3. On ne peut pas prescrire simultanément le spectre, le volume et la classe conforme. En effet, si on fixe le volume et la classe conforme, les valeurs propres ne peuvent pas être arbitrairement grandes (cf. [Fraser et Schoen 2011] et [Hassannezhad 2011]). Des obstructions semblables existent pour le laplacien usuel [El Soufi et Ilias 1986], le laplacien de Hodge en restriction aux formes différentielles de certains degrés [Jammes 2007; Jammes 2008] et l'opérateur de Dirac [Ammann 2003]. Le théorème 1.2 montre en revanche que même en fixant la classe conforme, on ne peut pas majorer le rapport $\sigma_{k} / \sigma_{l}$ pour $k>l$.

Remarque 1.4. On sait que la prescription de multiplicité est possible pour les opérateurs de Schrödinger en dimension $n \geq 3$ [Colin de Verdière 1986; 1987] et les opérateurs agissant sur les formes différentielles en dimension $n \geq 4$ [Jammes $2011 ; 2012$ ]. Mais ce problème n'est toujours pas résolu pour les formes différentielles en dimension 3, ni pour l'opérateur de Dirac, dont on ne sait actuellement prescrire le spectre que si les valeurs propres sont simples [Dahl 2005].

La principale difficulté consiste à prescrire la multiplicité des valeurs propres. On utilisera pour cela les techniques introduites dans [Colin de Verdière 1986] (voir [Jammes 2009] pour une présentation plus détaillée de ce sujet). Les principaux ingrédients sont des théorèmes de convergence spectrale (en particulier de convergence du spectre vers celui d'un domaine de la variété : théorème 3.8), un modèle de valeur propre multiple fourni par un laplacien combinatoire sur des graphes (paragraphe 4B).

La démonstration du théorème 1.2 échoue en dimension 2, entre autres à cause de l'invariance conforme de la norme $L^{2}$ du gradient d'une fonction. On va montrer que cette difficulté ne peut pas être contournée et qu'il existe en fait une obstruction à la prescription de multiplicité en dimension 2. La démonstration suit celles de S. Y. Cheng [1976] et G. Besson [1980] pour majorer la multiplicité des valeurs propres du laplacien.

Théorème 1.5. Sur toute surface riemannienne compacte orientable à bord $(M, g)$ de genre $\gamma$ et toute fonction strictement positive $\rho \in C^{0}(\partial M)$, la multiplicité de $\sigma_{k}(M, \rho, g)$ est majorée par $4 \gamma+2 k+1$. Si $M$ est non orientable et qu'on note l le nombre de composantes connexes de $\partial M$, alors la multiplicité de $\sigma_{k}(M, \rho, g)$ est majorée par $4 p+4 k+1$, où p est l'invariant topologique $1-\chi(M)-l$.

Remarque 1.6. Lors de la finalisation de cet article, la démonstration de bornes sur la multiplicité est apparu simultanément dans deux prépublications. A. Fraser et R. Schoen [2012] ont montré indépendamment le même théorème, avec une démonstration presque identique. Ils montrent aussi que la borne obtenue 
pour $\sigma_{1}\left(S^{1} \times[0,1]\right)$, à savoir 3, est optimale. Simultanément, M. Karpukhin, G. Kokarev, I. Polterovich ont démontré dans [Karpukhin et al. 2012] une de ces bornes avec des techniques différentes : ils montrent que la multiplicité de $\sigma_{k}$ est majorée par $2 p+2 k+1$ et $2 p+2 l+k$, que la surface soit orientable ou non.

Les bornes données par le théorème 1.5 sont les mêmes que celles obtenues par G. Besson pour les valeurs propres de laplacien. Il s'avère que le spectre de Steklov possède des rigidités supplémentaires qu'on va illustrer dans le cas du disque :

Théorème 1.7. Sur le disque $\mathbb{D}$, la multiplicité de $\sigma_{1}(\mathbb{D}, \rho, g)$ est au plus 2 et celle de $\sigma_{2}(\mathbb{D}, \rho, g)$ est au plus 3.

Remarque 1.8. En utilisant les résultats de [Colin de Verdière 1988], on peut facilement construire (par excision d'un petit disque sur la sphère) une métrique sur $\mathbb{D}$ telle que la première valeur propre non nulle du laplacien avec condition de Neumann (ou la seconde pour la condition de Dirichlet) soit de multiplicité 3 . En outre, la borne sur la multiplicité de $\sigma_{1}$ est optimale puisque pour la métrique canonique, toutes les valeurs propres non nulles sont doubles.

Remarque 1.9. L'article [Alessandrini et Magnanini 1994], qui traite de la multiplicité des $\sigma_{k}$ sur le disque, contient comme cas particulier le fait que la multiplicité de $\sigma_{1}$ est au plus 2 ; on en donnera une démonstration un peu plus directe. En revanche, la borne sur la multiplicité de $\sigma_{2}$ ne semble pas être apparue auparavant dans la littérature.

On va aussi montrer une autre borne sur la multiplicité dans le cas du disque, mais pour une variante du problème de Steklov, à savoir le problème de Steklov-Neumann. Ce problème est défini de la manière suivante : on partitionne le bord $\partial M$ en deux sous-variétés (pas nécessairement connexes) $\partial M=\partial M_{S} \cup \partial M_{N}$ et pour une fonction $\rho \in C^{0}\left(\partial M_{S}\right)$, on pose la condition $\partial f / \partial v=\sigma \rho f$ sur $\partial M_{S}$ et on demande à $f$ de vérifier la condition de Neumann sur $\partial M_{N}$ (voir paragraphe 2B pour plus de détails).

Théorème 1.10. Étant donnée une partition (non triviale) $\partial \mathbb{D}_{S} \cup \partial \mathbb{D}_{N}$ du bord du disque $\mathbb{D}$, la multiplicité de $\sigma_{k}(\mathbb{D}, \rho, g)$ pour le problème de Steklov-Neumann relativement à cette partition est au plus $k+1$.

Remarque 1.11. Pour le laplacien, les meilleures bornes connues sont asymptotiquement de l'ordre de $2 k$ quand $k \rightarrow+\infty$. Par exemple, pour le laplacien de Dirichlet sur le disque, il et montré dans [Hoffmann-Ostenhof et al. 1999] que la multiplicité de la $k$-ième valeur propre est au plus $2 k-3$. Dans [Karpukhin et al. 2012], la meilleure borne donnée pour le problème de Steklov sur le disque est $k+2$.

Remarque 1.12. Dans le cas particulier du problème hydrodynamique de ballottement (voir [Kopachevsky et Krein 2001] ou les rappels du paragraphe 2B), on sait que la première valeur propre non nulle est simple (cf. [Kozlov et al. 2004]). Il est conjecturé que les autres sont simples aussi, mais cette question reste ouverte.

Colin de Verdière a conjecturé que la multiplicité maximale de la deuxième valeur propre d'un opérateur de Schrödinger sur une surface $M$ est $\operatorname{Chr} M-1$, où Chr $M$ est le nombre chromatique de $M$, c'est-à-dire le nombre de sommets du plus grand graphe complet plongeable dans $M$. Comme la démonstration du théorème 1.2 repose sur des graphes plongées dans $M$ dont les sommets sont sur le bord de la variété, on peut envisager de transposer cette conjecture au problème de Steklov sous la forme suivante : 
Conjecture 1.13. Soit $M$ une surface compacte à bord $M$, et soit $\operatorname{Chr}(M, \partial M)$ le nombre de sommets du plus grand graphe complet qu'on peut plonger dans $M$ en plaçant les sommets sur $\partial M$. Alors la multiplicité maximale de $\sigma_{1}(M)$ est $\operatorname{Chr}(M, \partial M)-1$.

D'après ce qui précède, cette conjecture est vérifiée sur le disque $\mathbb{D}$ et le cylindre $S^{1} \times[0,1]$.

La section 2 rappellera quelques propriétés du spectre de Steklov et de l'opérateur Dirichlet-to-Neumann dont nous auront besoin. Nous montrerons dans la section 3 les théorèmes de convergence spectrale que nous utiliserons, et nous les appliquerons dans la section 4 pour démontrer le théorème 1.2. Enfin, la section 5 sera consacrée au cas de la dimension 2 et à la démonstration des théorèmes $1.5,1.7$ et 1.10.

\section{Le problème de Steklov}

2A. Définition du spectre de Steklov. On se donne une variété riemannienne $(M, g)$ compacte à bord telle que $\partial M$ soit $C^{1}$ par morceau (dans la suite, $g$ désignera indifféremment la métrique sur $M$ ou la métrique induite sur $\partial M$ ). Le problème des valeurs propres de Steklov consiste à résoudre l'équation

$$
\begin{cases}\Delta f=0 & \text { dans } M \\ \partial f / \partial v=\sigma \rho f & \text { sur } \partial M\end{cases}
$$

où $v$ est un vecteur unitaire sortant normal au bord et $\rho \in C^{0}(\partial M)$ un fonction densité fixée. L'ensemble des réels $\sigma$ solutions du problème forme un spectre discret positif noté

$$
0=\sigma_{0}(M, g, \rho)<\sigma_{1}(M, g, \rho) \leq \sigma_{2}(M, g, \rho) \leq \cdots .
$$

Le problème de Steklov, déjà étudié à la fin du XIXe siècle et au début du XXe (voir [Stekloff $1899 ; 1902$ ] et les références qui y sont données), apparaît dans divers problèmes physiques. Par exemple il permet de modéliser l'évolution d'une membrane libre dont la masse se concentre sur son bord, et il intervient dans certains problèmes de tomographie. On verra au paragraphe qui suit qu'il apparait aussi en hydrodynamique.

Notre principal outil sera la caractérisation variationnelle suivante du spectre de Steklov (cf. [Bandle 1980]) :

$$
\sigma_{k}(M, g, \rho)=\inf _{V_{k+1} \in H^{1}(M)} \sup _{f \in V_{k+1} \backslash\{0\}} \frac{\int_{M}|\mathrm{~d} f|^{2} \mathrm{~d} v_{g}}{\int_{\partial M} f^{2} \rho \mathrm{d} v_{g}},
$$

où $V_{k}$ parcours les sous-espaces de dimension $k$ de l'espace de Sobolev $H^{1}(M)$.

Il faut prendre garde au fait que $|f|^{2}=\int_{\partial M} f^{2} \rho \mathrm{d} v_{g}$ ne définit pas une norme de Hilbert sur $L^{2}(M)$ (elle est nulle sur les fonctions vérifiant la condition de Dirichlet). En revanche, on peut utiliser les techniques usuelles de min-max en considérant l'espace de Hilbert $L^{2}(\partial M)$ muni de la métrique $|\cdot|$ qu'on vient de définir, et la forme quadratique $Q(f)=\int_{M}|\mathrm{~d} \tilde{f}|^{2} \mathrm{~d} v_{g}$, où $\tilde{f}$ est le prolongement harmonique de $f$. Il sera parfois commode de redéfinir la forme quadratique $Q$ par

$$
Q(f)=\inf _{\substack{\left.\tilde{f} \in H^{1}(M) \\ \tilde{f}\right|_{\partial M}=f}} \int_{M}|\mathrm{~d} \tilde{f}|^{2} \mathrm{~d} v_{g} .
$$


Cette définition sera en particulier applicable dans les situations où on considère une métrique singulière sur $M$ (voir paragraphe 2C).

Dans le cas homogène, c'est-à-dire quand $\rho \equiv 1$, le spectre de Steklov est aussi connu comme étant le spectre de l'opérateur Dirichlet-to-Neumann, qu'on notera $\Lambda: C^{\infty}(\partial M) \rightarrow C^{\infty}(\partial M)$, défini comme suit : étant donné une fonction $f \in C^{\infty}(\partial M)$, on prolonge harmoniquement $f$ dans $M$ et on pose

$$
\Lambda f(x)=\frac{\partial f}{\partial v}(x) .
$$

Le spectre de $\Lambda$ est bien celui de $Q$ car pour une fonction harmonique, on a $\int_{M}|\mathrm{~d} f|^{2} \mathrm{~d} v_{g}=\int_{\partial M} f \frac{\partial f}{\partial \nu} \mathrm{d} v_{g}$. L'opérateur $\Lambda$ n'est pas un opérateur différentiel sur $\partial M$ (ce n'est même pas un opérateur local), mais c'est un opérateur pseudo-différentiel elliptique d'ordre 1 (cf. [Taylor 1996b, Chapter 7]). En particulier, nous utiliserons le fait qu'il vérifie une inégalité elliptique :

$$
\|f\|_{H^{1}(\partial M)}^{2} \leq c \int_{\partial M} f \Lambda f \mathrm{~d} v_{g}+c^{\prime}\|f\|_{L^{p}(\partial M)}^{2},
$$

où $p \in[1,+\infty]$, les constante $c, c^{\prime}$ dépendant de $p$ et de la métrique $g$ sur $M$ mais pas de $f$.

Pour finir, nous auront besoin d'une propriété d'unique prolongement des fonctions propres en dimension 2 :

Théorème 2.7. Soit $f$ une fonction propre du problème de Steklov sur une surface. Si $f$ s'annule sur un ouvert du bord, alors $f \equiv 0$.

Démonstration. Soit $I$ un intervalle du bord sur lequel $f$ s'annule. On peut déformer conformément la surface de manière à ce que $I$ devienne géodésique et que la métrique reste inchangée sur le reste du bord. Par invariance conforme de l'harmonicité et de la condition $\partial f / \partial v=0, f$ est toujours fonction propre. En notant $x$ un paramètre sur $I$, on a $\partial^{2} f / \partial x^{2}=0$, donc aussi $\partial^{2} f / \partial v^{2}=0$ puisque $f$ est harmonique. Enfin, comme $\partial f / \partial v=0$ sur $I$, on a aussi $\partial^{2} f / \partial x \partial v=0$ et donc le développement à l'ordre 2 de $f$ est nul le long de $I$.

Par conséquent, au voisinage d'un point de $I$, on peut prolonger $f$ par 0 en dehors de $M$ et obtenir une fonction $\tilde{f}$ qui est $C^{2}$ et vérifie $\Delta f=0$. Par unique prolongement des fonctions harmoniques, on a $f \equiv 0$ sur $M$.

2B. Le problème de Steklov-Neumann. Étant donné une variété compacte à bord $M$, on se donne un domaine (ou une union de domaines disjoints) à bord $C^{1}$ par morceaux de $\partial M$ qu'on notera $\partial M_{S}$, et on pose $\partial M_{N}=\partial M \backslash \partial M_{S}$. Si $\rho$ est une fonction sur $\partial M_{S}$, le problème de Steklov-Neumann se pose ainsi :

$$
\begin{cases}\Delta f=0 & \text { dans } M, \\ \partial f / \partial v=\sigma \rho f & \text { sur } \partial M_{S}, \\ \partial f / \partial v=0 & \text { sur } \partial M_{N},\end{cases}
$$

c'est-à-dire qu' on demande à la fonction harmonique $f$ de vérifier la condition de Neumann sur $\partial M_{N}$. On appellera respectivement bord de Steklov et bord de Neumann les ensembles $\partial M_{S}$ et $\partial M_{N}$. Les solutions de ce problème interviennent dans l'étude du phénomène hydrodynamique de ballottement (sloshing 
problem) : si on considère un fluide parfait incompressible contenu dans un récipient $M$ avec une surface libre $\partial M_{S}$, les petites oscillations périodiques du fluide correspondent aux solutions de (2.8) pour une fonction $\rho$ constante (voir, par exemple, [Kopachevsky et Krein 2001]).

Le problème de Steklov-Neumann possède un spectre discret et positif qu'on notera

$$
0=\sigma_{0}\left(M, \partial M_{S}, g, \rho\right)<\sigma_{1}\left(M, \partial M_{S}, g, \rho\right) \leq \sigma_{2}\left(M, \partial M_{S}, g, \rho\right) \leq \cdots
$$

Le spectre de Steklov-Neumann possède la même caractérisation variationnelle que le spectre de Steklov, à condition de restreindre l'intégrale sur le bord au bord de Steklov :

$$
\sigma_{k}\left(M, \partial M_{S}, g, \rho\right)=\inf _{V_{k+1} \in H^{1}(M)} \sup _{f \in V_{k+1} \backslash\{0\}} \frac{\int_{M}|\mathrm{~d} f|^{2} \mathrm{~d} v_{g}}{\int_{\partial M_{S}} f^{2} \rho \mathrm{d} v_{g}},
$$

où $V_{k}$ parcours les sous-espaces de dimension $k$ de $H^{1}(M)$.

L'opérateur Dirichlet-to-Neumann est bien défini sur $\partial M_{S}$ en considérant des fonctions harmoniques vérifiant la condition de Neumann sur $\partial M_{N}$ et vérifie toujours l'inégalité elliptique (2.6).

On aura besoin du fait que si on se donne une fonction $f$ sur $\partial M_{S}$ et qu'on la prolonge en une fonction harmonique (toujours notée $f$ ), sa norme $L^{2}$ sur $\partial M_{N}$ est contrôlée par sa norme sur $\partial M_{S}$, c'est-à-dire qu'il existe une constante $c>0$ ne dépendant que de $g$ et $\rho$ telle que $\int_{\partial M_{N}} f^{2} \leq c \int_{\partial M_{S}} f^{2} \rho$. Cela découle du fait que la norme $L^{2}\left(\partial M_{N}\right)$ de $f$ est contrôlée par sa norme $H^{1 / 2}(M)$, elle même contrôlée par sa norme $L^{2}\left(\partial M_{S}\right)$ (cf. [Taylor 1996a, §4.4]).

On utilisera aussi un bref usage du spectre de Steklov-Dirichlet, défini en considérant des fonctions harmoniques qui vérifient la condition $f=0$ sur $\partial M \backslash \partial M_{S}$. La propriété de ce spectre qui nous intéressera est qu'il est strictement positif (cf. [Agranovich 2006]).

2C. Fonctions harmoniques et métriques singulières. Dans la section suivante, on aura à manipuler des métriques discontinues. Si $U$ est un domaine de $(M, g)$ et $\varepsilon \in[0,1]$ un réel fixé, elles seront de la forme

$$
\begin{cases}g_{\varepsilon}=\varepsilon^{2} g & \text { sur } U, \\ g_{\varepsilon}=g & \text { sur } M \backslash U .\end{cases}
$$

Comme les normes $L^{2}$ et de Sobolev pour les métriques $g$ et $g_{\varepsilon}$ sont équivalentes, la théorie spectrale de la forme quadratique $\|\mathrm{d} \cdot\|_{g_{\varepsilon}}^{2}$ sur $H^{1}(M)$ est donc similaire à celle de $\|\mathrm{d} \cdot\|_{g}^{2}$. On peut donc définir le prolongement harmonique d'une fonction $f \in C^{\infty}(\partial M)$ comme étant le prolongement minimisant cette forme quadratique. Cette définition est cohérente avec celle de la forme quadratique $Q$ donnée par (2.4).

Un tel prolongement minimisera en particulier $\|\mathrm{d} \cdot\|_{g_{\varepsilon}}^{2}$ en restriction à chacun des domaines $U$ et $M \backslash U$, il sera donc harmonique au sens usuel sur ces deux domaines.

Les mêmes remarques s'appliquent au problème de Steklov-Neumann.

\section{Théorèmes de convergence spectrale}

3A. Rappels. Dans cette section, nous allons montrer plusieurs théorèmes de convergence spectrale dont nous aurons besoin pour prescrire le spectre de Steklov. Nous utiliserons pour cela les techniques développées dans [Colin de Verdière 1986]. Pour prescrire la multiplicité des valeurs propres, il nous 
faudra montrer la convergence des espaces propres, et nous aurons aussi besoin d'une certaine uniformité de la convergence, nous reprendrons pour cela les notations de Colin de Verdière :

Soit $E_{0}$ et $E_{1}$ sont deux sous-espaces vectoriels de même dimension $N$ d'un espace de Hilbert, munis respectivement des formes quadratiques $q_{0}$ et $q_{1}$. Si $E_{0}$ et $E_{1}$ sont suffisamment proches, il existe une isométrie naturelle $\psi$ entre les deux (voir la section I de [Colin de Verdière 1986] pour les détails de la construction), on définit alors l'écart entre $q_{0}$ et $q_{1}$ par $\left\|q_{1} \circ \psi-q_{0}\right\|$. Pour deux formes quadratiques $Q_{0}$ et $Q_{1}$ sur l'espace de Hilbert, on appellera $N$-écart spectral entre $Q_{0}$ et $Q_{1}$ l'écart entre les deux formes quadratiques restreintes à la somme des espaces propres associés aux $N$ premières valeurs propres. Si cet écart est petit, alors les $N$ premières valeurs propres de $Q_{0}$ et leurs espaces propres sont proches de ceux de $Q_{1}$.

On veut montrer que la convergence spectrale est uniforme pour une certaine famille de spectres limites. D'après Colin de Verdière, on dira donc qu'une forme quadratique vérifie l'hypothèse (*) si ses valeurs propres vérifient

$$
\lambda_{1} \leq \cdots \leq \lambda_{N}<\lambda_{N}+\eta \leq \lambda_{N+1} \leq M
$$

pour un entier $N$ et des réels $\eta, M>0$ fixés une fois pour toutes. Dans les énoncés suivants, les constantes $N, M$ et $\eta$ auront ces valeurs préalablement fixées.

Lemme 3.1 [Colin de Verdière 1986, théorème I.7]. Soit $Q$ une forme quadratique positive sur un espace de Hilbert $\mathscr{H}$ dont le domaine admet la décomposition $Q$-orthogonale $\operatorname{dom}(Q)=\mathscr{H}_{0} \oplus \mathscr{H}_{\infty}$. Pour tout $\varepsilon>0$, il existe une constante $C(\eta, M, N, \varepsilon)>0$ (grande) telle que si $Q_{0}=\left.Q\right|_{\mathscr{H}_{0}}$ vérifie l'hypothèse (*) et si $Q(x) \geq C|x|^{2}$ pour tout $x \in \mathscr{H}_{\infty}$, alors $Q$ et $Q_{0}$ ont un $N$-écart spectral inférieur à $\varepsilon$.

Lemme 3.2 [ibid., théorème I.8]. Soit $(\mathscr{H},|\cdot|)$ un espace de Hilbert muni d'une forme quadratique positive $Q$. On se donne en outre une suite de métriques $|\cdot|_{n}$ sur $\mathcal{H}$ et une suite de formes quadratiques $Q_{n}$ de même domaine que $Q$ telles que:

(i) il existe $C_{1}, C_{2}>0$ tels que $C_{1}|x| \leq|x|_{n} \leq C_{2}|x|$ pour tout $x \in \mathscr{H}$;

(ii) $|x|_{n} \rightarrow|x|$ pour tout $x \in \operatorname{dom}(Q)$;

(iii) $Q(x) \leq Q_{n}(x)$ pour tout $x \in \operatorname{dom}(Q)$;

(iv) $Q_{n}(x) \rightarrow Q(x)$ pour tout $x \in \operatorname{dom}(Q)$.

Si $Q$ vérifie l'hypothèse (*), alors à partir d'un certain rang (dépendant de $\eta, M$ et $N$ ), $Q$ et $Q_{n}$ ont un $N$-écart spectral inférieur à $\varepsilon$.

Remarque 3.3. Comme on l'a remarqué dans [Jammes 2011], dans le lemme 3.2, on peut affaiblir l'hypothèse $C_{1}|x| \leq|x|_{n} \leq C_{2}|x|$ en $C_{1}|x| \leq|x|_{n} \leq C_{2}|x|+\varepsilon_{n} Q_{n}(x)^{1 / 2}$ avec $\varepsilon_{n} \rightarrow 0$, la démonstration restant exactement la même (on peut aussi remplacer $Q_{n}$ par $Q$ dans cette dernière inégalité). En particulier, il n'est pas nécessaire que l'espace de Hilbert $(\mathscr{H},|\cdot|)$ soit complet pour $|\cdot|_{n}$.

Remarque 3.4. On peut aussi remplacer l'hypothèse $x \in \operatorname{dom}(Q) \Rightarrow Q(x) \leq Q_{n}(x)$ par $Q(x) \leq M \Rightarrow$ $Q(x) \leq\left(1+\varepsilon_{n}\right) Q_{n}(x)$ avec $\varepsilon_{n} \rightarrow 0$. 
Remarque 3.5. Pour déduire la convergence du spectre et des espaces propres de la convergence des formes quadratiques, on doit en principe se ramener à une norme de Hilbert fixe. Ça ne sera pas nécessaire dans la suite car les étapes de la démonstration où la norme varie seront traitées à l'aide du lemme 3.2.

3B. Densité et convergence de spectre. Notre premier résultat de convergence sera de montrer qu'avec une densité fixée sur le bord, on peut déformer conformément la métrique de manière à faire tendre le spectre de Steklov vers le spectre correspondant à une autre densité. On peut en outre faire tendre la métrique déformée vers la métrique initiale dans l'intérieur de la variété. On se restreindra au cas où la densité initiale est plus petite que la densité du spectre limite, ce qui sera suffisant pour les applications dans la section 4 .

Théorème 3.6. Soit $(M, g)$ une variété riemannienne compacte à bord, et $\rho, \bar{\rho} \in C^{0}(\partial M)$ deux fonctions sur le bord de $M$ telles que $\bar{\rho} \geq \rho$.

Il existe une famille $g_{\varepsilon}$ de métriques conformes à $g$ sur $M$ telle que:

(i) $\sigma_{k}\left(M, g_{\varepsilon}, \rho\right)$ tend vers $\sigma_{k}(M, g, \bar{\rho})$ quand $\varepsilon \rightarrow 0$ pour tout $k \geq 0$, avec convergence des espaces propres.

(ii) $g_{\varepsilon}=(\bar{\rho} / \rho)^{2 /(n-1)} g$ sur $\partial M$.

(iii) $g_{\varepsilon}$ tend vers $g$ uniformément sur tout compact dans l'intérieur de $M$.

En outre, si les $\sigma_{k}(M, g, \bar{\rho})$ vérifient l'hypothèse $(*)$, alors le $N$-écart spectral entre $\left(M, g_{\varepsilon}, \rho\right)$ et $(M, g, \bar{\rho})$ tend vers 0 .

Démonstration. On définit une famille $h_{\varepsilon} \in C^{\infty}(M)$ de facteurs conformes de la manière suivante : on fixe $h_{\varepsilon}(x)=(\bar{\rho} / \rho)^{1 /(n-1)}$ pour $x \in \partial M$ et on étend $h_{\varepsilon}$ de manière lisse de sorte que la famille $\left(h_{\varepsilon}\right)$ tende simplement vers 1 dans l'intérieur de $M$, et uniformément sur tout compact ne rencontrant pas le bord. On pose alors $g_{\varepsilon}=h_{\varepsilon}^{2} g$ pour tout $\varepsilon$.

La famille de métriques $g_{\varepsilon}$ induit les familles de normes et de formes quadratiques

$$
Q_{\varepsilon}(f)=\inf _{\substack{\tilde{f} \in H^{1}(M) \\ \tilde{f} \mid \partial M=f}} \int_{M} h_{\varepsilon}^{n-2}|\mathrm{~d} \tilde{f}|^{2} \mathrm{~d} v_{g} \quad \text { et } \quad|f|_{\varepsilon}=\int_{\partial M} f^{2} \bar{\rho} \mathrm{d} v_{g} .
$$

Comme $\bar{\rho} \geq \rho$, on peut choisir une suite $\left(h_{\varepsilon}\right)$ décroissante, les suites $Q_{\varepsilon}$ et $|\cdot|_{\varepsilon}$ vérifient alors les hypothèses du lemme 3.2 , ce qui suffit pour conclure.

3C. Convergence vers le spectre d'un domaine. Le second théorème consiste à faire converger le spectre de Steklov d'une variété à bord $M$ vers le spectre de Steklov-Neumann d'un domaine $U$ de $M$, avec la condition de Steklov sur $\partial U_{S}=\partial U \cap \partial M$ et la condition de Neumann sur le reste du bord de $U$. Ce résultat étend au spectre de Steklov de théorèmes analogues concernant le laplacien agissant sur les fonctions [Colin de Verdière 1986] et sur les formes différentielles [Jammes 2011].

Théorème 3.8. Soit $\left(M^{n}, g\right)$ une variété riemannienne compacte à bord de dimension $n \geq 3, \rho \in C^{\infty}(\partial M)$ et $U$ un domaine de $M$ à bord $C^{1}$ par morceau tel que $\partial U_{S}=\partial U \cap \partial M$ soit non vide. Il existe une famille $g_{\varepsilon}$ de métriques sur $M$ conformes à $g$ telle que: 
(i) $g=g_{\varepsilon}$ en restriction à $U$.

(ii) $\operatorname{Vol}\left(M, g_{\varepsilon}\right) \rightarrow \operatorname{Vol}(U, g)$ quand $\varepsilon \rightarrow 0$.

(iii) $\sigma_{k}\left(M, \rho, g_{\varepsilon}\right) \rightarrow \sigma_{k}\left(U, \partial U_{S},\left.\rho\right|_{\partial U_{S}},\left.g\right|_{U}\right)$ quand $\varepsilon \rightarrow 0$ pour tout $k \geq 0$, avec convergence des espaces propres.

En outre, si les $\sigma_{k}\left(U, \partial U_{S},\left.\rho\right|_{\partial U_{S}},\left.g\right|_{U}\right)$ vérifient l'hypothèse $(*)$, alors le $N$-écart spectral entre $\left(M, \rho, g_{\varepsilon}\right)$ et $\left(U, \partial U_{S},\left.\rho\right|_{\partial U_{S}},\left.g\right|_{U}\right)$ tend vers 0 .

Démonstration. La démonstration est similaire à celle du théorème III.1 de [Colin de Verdière 1986] et passe par l'intermédiaire, pour un réel $\eta>0$ petit donné, de la métrique singulière $g_{\eta}$ définie par $g_{\eta}=g$ sur $U$ et $g_{\eta}=\eta^{2} g$ sur $M \backslash U$. Elle se déroule en deux étapes : d'abord, on montre la convergence spectre pour la famille de métriques singulières, puis on approche ces métriques singulières par des métriques lisses. On conclut en se donnant, pour un $\varepsilon>0$ donné, une métrique $g_{\eta}$ tel que l'écart spectral avec le spectre de $(U, g)$ soit inférieur à $\varepsilon$, puis une métrique lisse $g_{\varepsilon}$ tel que l'écart spectral avec $g_{\eta}$ soit lui aussi inférieur à $\varepsilon$.

On fera souvent appel à la forme quadratique définie en (2.4), en particulier quand on manipule des métriques singulières.

Étape 1 . Un réel $\eta>0$ étant donné, la métrique $g_{\eta}$ induit sur $L^{2}(\partial M)$ la forme quadratique

$$
Q_{\eta}(f)=\inf _{\left.\tilde{f}\right|_{\partial M}=f}\left(\int_{U}|\mathrm{~d} \tilde{f}|^{2} \mathrm{~d} v_{g}+\eta^{n-2} \int_{M \backslash U}|\mathrm{~d} \tilde{f}|^{2} \mathrm{~d} v_{g}\right)
$$

et la norme $|f|_{g_{\eta}}=\int_{\partial U_{S}} f^{2} \rho \mathrm{d} v_{g}+\eta^{(n-1)} \int_{\partial M \backslash \partial U_{S}} f^{2} \rho \mathrm{d} v_{g}$. On va utiliser le lemme 3.1 pour se ramener à un sous-domaine de la forme quadratique puis appliquer le lemme 3.2.

En notant $\mathscr{H}$ le domaine de la forme quadratique $Q_{\eta}$, on définit l'espace $\mathscr{H}_{\infty}=\left\{f \in \mathscr{H},\left.f\right|_{\partial U_{S}}=0\right\}$ et on note $\mathcal{H}_{0}$ son orthogonal pour la forme quadratique $Q_{\eta}$. Pour appliquer le lemme 3.1, on doit minorer la forme quadratique $Q_{\eta}$ sur $\mathscr{H}_{\infty}$ en fonction de $|\cdot|_{\eta}$. Si $f \in \mathscr{H}_{\infty}$, alors

et

$$
|f|_{\eta}^{2}=\eta^{(n-1)} \int_{\partial M \backslash \partial U_{S}} f^{2} \rho \mathrm{d} v_{g}=\eta^{(n-1)}|f|^{2}
$$

$$
Q_{\eta}(f) \geq \eta^{n-2} \inf _{\left.\tilde{f}\right|_{\partial M}=f} \int_{M}|\mathrm{~d} \tilde{f}|^{2} \mathrm{~d} v_{g}=\eta^{n-2} Q(f) .
$$

On est donc ramené à l'étude du spectre de la forme quadratique $Q$ associée à la métrique initiale $g$ en restriction à l'espace $\mathscr{H}_{\infty}$, c'est-à-dire à minorer le spectre de Steklov sur $M$ avec condition de Dirichlet sur $\partial U_{S}$. Comme 0 n'est pas dans le spectre de Steklov-Dirichlet (cf. paragraphe 2B), il existe une constante $c>0$ telle que $Q(f) /|f|^{2}>c$ pour tout $f \in \mathscr{H}_{\infty}$. Par conséquent, $Q_{\eta}(f) /|f|_{\eta} \geq c \cdot \eta^{-2}$ pour tout $f \in \mathscr{H}_{\infty}$. Si $\eta$ est suffisamment petit, on peut donc appliquer le lemme 3.1 et en déduire que le spectre pour la métrique $g_{\eta}$ est proche du spectre de $Q_{\eta}$ restreint à $\mathcal{H}_{0}$.

Il reste à montrer que la limite du spectre de $\left.Q_{\eta}\right|_{\mathscr{H}_{0}}$ est le spectre de Steklov-Neumann du domaine $U$. On utilisera pour cela le lemme 3.2. Puisque $\mathscr{H}_{0}$ est défini comme le $Q_{\eta}$-orthogonal des fonctions de $\partial M$ nulles sur $\partial U_{S}$, une fonction de $\mathscr{H}_{0}$ est entièrement déterminée par sa restriction à $\partial U_{S}$. Plus précisément, 
parmi les fonctions $f$ dont la valeur sur $\partial U_{S}$ est fixée, celle qui est dans $\mathscr{H}_{0}$ est celle minimise la forme quadratique $Q_{\eta}$. C'est donc la restriction au bord du prolongement harmonique (tel qu'on l'a défini au paragraphe 2C) de $f_{\partial U_{S}}$ avec condition de Neumann sur $\partial M \backslash \partial U_{S}$. Dans la suite, on identifiera souvent une fonction sur $\partial U_{S}$ avec le prolongement ainsi défini.

La norme $|\cdot|_{\eta}$ converge en décroissant vers la norme $|\cdot|$ définie par $|f|=\int_{\partial U_{S}} f^{2} \rho \mathrm{d} v_{g}$. L'hypothèse (ii) et la première inégalité de l'hypothèse (i) du lemme 3.2 sont donc satisfaites. Les hypothèses (iii) et (iv) sont vérifiées pour les mêmes raisons.

Il reste à montrer que la deuxième inégalité de l'hypothèse (i) est vérifiée. Pour cela, on doit majorer $\int_{\partial M \backslash \partial U_{S}} f^{2} \rho \mathrm{d} v_{g}$. Notons $\tilde{f}$ le prolongement de $\left.f\right|_{\partial U_{S}}$ qui est harmonique au sens du paragraphe $2 \mathrm{C}$, c'est-à-dire que

$$
Q_{\eta}(f)=\int_{U}|\mathrm{~d} \tilde{f}|^{2} \mathrm{~d} v_{g}+\eta^{n-2} \int_{M \backslash U}|\mathrm{~d} \tilde{f}|^{2} \mathrm{~d} v_{g} .
$$

Comme on l'a remarqué au paragraphe 2B, puisque $\tilde{f}$ est harmonique sur $M \backslash U$ avec condition de Neumann sur $\partial M \backslash \partial U_{S}$, la norme $L^{2}\left(\partial M \backslash \partial U_{S}, \rho\right)$ de $\tilde{f}$ est contrôlée par sa norme $L^{2}$ sur $\partial U \backslash \partial U_{S}$, c'est-à-dire que

$$
\int_{\partial M \backslash \partial U_{S}} \tilde{f}^{2} \rho \mathrm{d} v_{g} \leq c_{1} \int_{\partial U \backslash \partial U_{S}} \tilde{f}^{2} \rho \mathrm{d} v_{g} .
$$

Notons que la constante $c_{1}$ est invariante par homothétie, donc indépendante de $\eta$, à condition de considérer sur $\partial(M \backslash U)$ la métrique induite par la métrique de $M \backslash U$. En considérant la métrique $g_{\eta}$ sur $\partial M \backslash \partial U_{S}$ et la métrique $g$ sur $\partial U \backslash \partial U_{S}$ on obtient

$$
\int_{\partial M \backslash \partial U_{S}} \tilde{f}^{2} \rho \mathrm{d} v_{g_{\eta}} \leq \eta^{n-1} c_{1} \int_{\partial U \backslash \partial U_{S}} \tilde{f}^{2} \mathrm{~d} v_{g}
$$

On majore le membre de droite à l'aide de l'inégalité elliptique de l'opérateur Dirichlet-to-Neumann sur $\partial U$.

$$
\int_{\partial U \backslash \partial U_{S}} \tilde{f}^{2} \mathrm{~d} v_{g} \leq\|f\|_{L^{2}(\partial U)}^{2} \leq\|f\|_{H^{1}(\partial U)}^{2} \leq c_{2} \int_{\partial U} f \frac{\partial f}{\partial \nu} \mathrm{d} v_{g}=c_{2} \int_{U}|\mathrm{~d} \tilde{f}|^{2} \mathrm{~d} v_{g} \leq c_{2} Q_{\eta}(f) .
$$

On a donc finalement $|f|_{\eta}^{2} \leq|f|^{2}+\eta^{n-1} c_{1} c_{2} Q_{\eta}(f)$ ce qui permet d'appliquer le lemme 3.2 et la remarque 3.3 .

Étape 2. On doit montrer que pour tout $\eta>0$, le spectre de $Q_{\eta}$ peut être approché par le spectre de métriques lisses conformes à $g$.

Le paramètre $\eta$ état fixé, on définit une suite de facteurs conformes $h_{i}$ tels que la suite $\left(h_{i}\right)$ converge en décroissant vers la fonction $\chi_{U}+\eta \chi_{M \backslash U}$ et on pose $g_{i}=h_{i}^{2} g$. Les suites de norme de Hilbert $|\cdot|_{i}$ et de formes quadratiques $Q_{i}$ associées à $g_{i}$ convergent vers $|\cdot|_{\eta}$ et $Q_{\eta}$ en vérifiant les hypothèses du lemme 3.2, ce qui assure la convergence du spectre et des espaces propres.

3D. Convergence vers le spectre du bord. Enfin, nous allons montrer qu'on peut faire tendre le spectre de Steklov homogène (c'est-à-dire que $\rho \equiv 1$ ) d'une variété à bord $M$ vers le spectre du laplacien sur $\partial M$, la métrique sur $\partial M$ restant homothétique à la métrique initiale. Bien que ce théorème ne soit pas 
indispensable pour démontrer le théorème 1.2, on peut l'utiliser si la dimension de $\partial M$ est au moins 3. Il semble aussi intéressant en lui-même et fournit un exemple d'application du théorème démontré au paragraphe précédent.

Théorème 3.12. Soit $\left(M^{n}, g\right)$ une variété riemannienne compacte à bord de dimension $n \geq 3$. Il existe une famille $g_{\varepsilon}$ de métriques sur $M$ conformes à $g$ et homothétiques à $g$ le long de $\partial M$ telle que pour tout $k \geq 0$ on ait $\sigma_{k}\left(M, g_{\varepsilon}\right) \rightarrow \lambda_{k}(M, g)$ quand $\varepsilon \rightarrow 0$.

En outre, si les $\lambda_{k}(M, g)$ vérifient l'hypothèse $(*)$, alors le $N$-écart spectral entre le spectre de Steklov de $\left(M, g_{\varepsilon}\right)$ et le spectre du laplacien de $(\partial M, g)$ tend vers 0 .

Démonstration. Le principe de la démonstration consiste à se ramener au cas d'un voisinage collier du bord (avec la condition mixte Steklov-Neumann) en utilisant le théorème 3.8. On va procéder en trois étapes : d'abord montrer la convergence du spectre d'un voisinage collier de $\partial M$ muni d'une métrique produit, puis traiter le cas de la restriction de métrique $g$ à ce voisinage collier, et enfin montrer la convergence du spectre de $M$.

Étape 1 . On va déterminer l'asymptotique (quand $\eta \rightarrow 0$ ) du spectre de la variété produit $\partial M \times[0, \eta]$ pour un métrique produit, avec la condition de Steklov sur $\partial M \times\{0\}$ et la condition de Neumann sur $\partial M \times\{\eta\}$.

On peut déduire ce spectre du spectre de Steklov de $\partial M \times[0,2 \eta]$ (avec condition de Steklov sur les deux bords) par symétrie : en effet, on peut partitionner les valeurs propres de $\partial M \times[0,2 \eta]$ en deux, selon que les fonctions propres sont symétriques ou antisymétriques. Ces fonctions propres vérifient la condition de Neumann sur $\partial M \times\{\eta\}$ dans le premier cas et la condition de Dirichlet dans le second cas. Le spectre de $\partial M \times[0, \eta]$ avec condition mixte est donc le spectre de Steklov de $\partial M \times[0,2 \eta]$ restreint aux fonctions symétriques.

Le spectre de Steklov de $\partial M \times[0,2 \eta]$ a été calculé explicitement dans lemme 6.1 de [Colbois et al. 2011] en fonction du spectre du laplacien sur $\partial M:$ si $\partial M$ est de volume 1 et si on note $\lambda_{k}$ ses valeurs propres et $u_{k}$ ses fonctions propres, alors le spectre non nul de $\partial M \times[0,2 \eta]$ restreint aux fonctions symétriques est $\sqrt{\lambda_{k}} \tanh \left(\eta \sqrt{\lambda_{k}}\right)$ les fonctions propres associées étant $\cosh \left(\sqrt{\lambda_{k}} t\right) u_{k}(x)$, où $x$ désigne un point de $\partial M$ et $t$ la coordonnée sur l'intervalle. Le spectre de Steklov-Neumann de $\partial M \times[0, \eta]$ se comporte donc asymptotiquement comme $\eta \lambda_{k}$ quand $\eta \rightarrow 0$. En pratiquant une homothétie sur $\partial M \times[0, \eta]$, on peut donc faire tendre son spectre vers $\lambda_{k}$. On peut facilement vérifier à l'aide de l'expression des fonctions propres qu'il y a bien convergence des espaces propres.

Étape 2. Étant donnée la variété à bord $(M, g)$ et un réel $\eta>0$ petit, on considère le $\eta$-voisinage collier $M_{\eta}$ de $\partial M$, c'est-à-dire que $M_{\eta}=\{x \in M, d(x, \partial M) \leq \eta\}$. Pour $\eta$ suffisamment petit, $M_{\eta}$ est difféomorphe au produit de $\partial M$ avec un intervalle. On considère alors le problème de Steklov-Neumann sur $M_{\eta}$ comme dans l'étape 1 .

Quand $\eta$ tend vers 0 , la métrique $g$ restreinte à $M_{\eta}$ est de plus en plus proche d'une métrique produit. Plus précisément, il existe une famille de réels $\tau_{\eta}>1$ telle que $\tau_{\eta} \rightarrow 1$ quand $\eta \rightarrow 0$ et $1 / \tau_{\eta} g_{\eta} \leq\left. g\right|_{M_{\eta}} \leq \tau_{\eta} g_{\eta}$, où $g_{\eta}$ désigne la métrique produit sur $\partial M \times[0, \eta]$. Comme la constante $\tau_{\eta}$ contrôle aussi l'écart entre les normes de Hilbert et les formes quadratiques pour le problème de Steklov-Neumann $\operatorname{sur}\left(M_{\eta}, g\right)$ et 
$\left(M_{\eta}, g_{\eta}\right)$, on peut appliquer le lemme 3.2 et la remarque 3.4 pour obtenir la convergence spectrale comme dans l'étape 1 .

Étape 3. Pour pouvoir conclure, il on aura besoin de faire tendre le spectre de Steklov de $M$ vers celui de $M_{\eta}$ en restant dans la classe conforme de $g$. On va utilise pour cela le théorème 3.8 :

Un réel $\varepsilon>0$ étant donné, on choisit $\eta>0$ et un rapport d'homothétie $r_{\eta}>0$ tels que le spectre de $\left(M_{\eta}, r_{\eta}^{2} g\right)$ soit $\varepsilon$-proche de celui de $(\partial M, g)$. Puis, en appliquant le théorème 3.8 avec $U=M_{\eta}$, on obtient une métrique $g_{\eta}^{\prime}$ sur $M$ tel que le spectre de $\left(M, g_{\eta}^{\prime}\right)$ soit $\varepsilon$-proche de celui de $\left(M_{\eta}, r_{\eta}^{2} g\right)$. Quand $\varepsilon$ tend vers 0 , on a ainsi convergence du spectre et des espaces propres de $\left(M, \rho, g_{\varepsilon}\right)$ vers ceux du laplacien sur $(\partial M, g)$.

\section{Prescription du spectre}

4A. L'hypothèse de transversalité d'Arnol'd. Pour prescrire la multiplicité des valeurs propres de Steklov nous utilisons, selon la méthode introduite par Colin de Verdière, trois ingrédients : les théorèmes de convergence spectrale démontrés dans les sections précédentes, des modèles de valeurs propres multiples déjà connus et une propriété de stabilité vérifiée par ces modèles. Nous allons commencer par rappeler cette dernière. On verra au paragraphe suivant comment utiliser des graphes complets comme modèles de spectre avec multiplicité.

On suppose qu'on a une famille d'opérateurs $\left(P_{a}\right)_{a \in B^{k}}$, où $B^{k}$ est la boule unité de $\mathbb{R}^{k}$ (en pratique, $P_{a}$ est l'opérateur Dirichlet-to-Neumann associé à une métrique $g_{a}$ ), tels que $P_{0}$ possède une valeur propre $\lambda_{0}$ d'espace propre $E_{0}$ et de multiplicité $N$. Pour les petites valeurs de $a, P_{a}$ possède des valeurs propres proches de $\lambda_{0}$ dont la somme des espaces propres est de dimension $N$. Comme dans la définition de l'écart spectral, on identifie cette somme à $E_{0}$ et on note $q_{a}$ la forme quadratique associée à $P_{a}$ transportée sur $E_{0}$.

Définition 4.1 [Colin de Verdière 1988]. On dit que $\lambda_{0}$ vérifie l'hypothèse de transversalité d'Arnol'd si l'application $\Psi: a \mapsto q_{a}$ de $B^{k}$ dans $2\left(E_{0}\right)$ est essentielle en 0 , c'est-à-dire qu'il existe $\varepsilon>0$ tel que si $\Phi: B^{k} \rightarrow 2\left(E_{0}\right)$ vérifie $\|\Psi-\Phi\|_{\infty} \leq \varepsilon$, alors il existe $a_{0} \in B^{k}$ tel que $\Phi\left(a_{0}\right)=q_{0}$.

Une propriété cruciale est que si $\Phi$ provient d'une famille $\left(P_{a}^{\prime}\right)$ d'opérateurs, alors $\lambda_{0}$ est valeur propre de $P_{a_{0}}^{\prime}$ de multiplicité $N$ et vérifie la même propriété de transversalité, ce qui justifie qu'on parle de stabilité de la multiplicité. Comme remarqué par Colin de Verdière, on peut généraliser cette définition à une suite finie de valeurs propres.

4B. Voisinages tubulaires de graphes. Colin de Verdière a montré qu'un graphe complet muni d'un laplacien combinatoire et d'une métrique appropriée possède une (ou plusieurs) valeur propre multiple vérifiant la propriété de transversalité d'Arnol'd. Dans ce paragraphe, nous allons utiliser ce résultat pour construire une variété dont on prescrit le début du spectre Steklov-Neumann avec multiplicité.

On note $\Gamma$ un graphe fini, $S$ l'ensemble de ses sommets et $A$ l'ensemble de ses arêtes. On se donne une métrique sur ce graphe en associant à chaque arête $a_{i} \in A$, une longueur $l_{i}>0$. Le laplacien combinatoire sur $\Gamma$ est l'opérateur agissant sur les fonctions $S \rightarrow \mathbb{R}$ induit par la forme quadratique 
$q(f)=\sum_{a_{i} \in A} l_{i} \mathrm{~d}_{a_{i}}(f)^{2}$, avec $\mathrm{d}_{a}(f)=(f(x)-f(y)) / l_{i}, x$ et $y$ étant les extrémités de l'arête $a_{i}$. L'espace des fonctions $\mathbb{R}^{S}$ sur les sommets est muni de sa structure euclidienne canonique.

On utilise alors le résultat suivant :

Théorème 4.2 [Colin de Verdière 1988, §4]. Étant donné une suite $0=\lambda_{0}<\lambda_{1} \leq \lambda_{2} \leq \cdots \leq \lambda_{N}$, il existe une métrique sur le graphe complet à $N+1$ sommets dont le spectre est la suite $\left(\lambda_{i}\right)$. De plus, ce spectre vérifie la propriété de transversalité d'Arnol'd.

Il reste à construire une famille de variétés dont le début du spectre ressemble à celui d'un graphe complet. Ces variétés, qu'on notera $\Omega_{\varepsilon}$, seront localement des domaines euclidiens. Le graphe $\Gamma$ sera plongeable isométriquement dans $\Omega_{\varepsilon}$, les sommets étant situés sur le bord de Steklov de la variété (construire $\Omega_{\varepsilon}$ comme étant globalement un domaine euclidien nécessiterait d'imposer des contraintes sur les longueurs $l_{i}$, ce qu'on veut éviter).

Plus précisément, deux réels $c>0$ et $\varepsilon$ étant fixé, on se donne pour chaque sommet $s_{j} \in S$ une demi-boule $B_{j}$ de rayon $c \varepsilon$ (dans la suite, utilisera l'indice $j$ pour les sommets du graphe et on réservera l'indice $i$ pour les arêtes). La constante $c$ sera fixée plus loin. Pour chaque arête $a_{i} \in A$, on se donne ensuite un cylindre $C_{i}$ de rayon $\varepsilon$ et de longueur $l_{i}-2 c \varepsilon$. Si on note $j$ et $j^{\prime}$ les indices des sommets extrémités de l'arête $a_{i}$, et qu'on place les boules $B^{n-1}$ qui bordent le cylindre $C_{i}$ tangentiellement aux demi-boules $B_{j}$ et $B_{j^{\prime}}$, on peut plonger isométriquement l'arête $a_{i}$ dans la réunion de $C_{i}, B_{j}$ et $B_{j^{\prime}}$,en identifiant les sommets $s_{j}$ et $s_{j^{\prime}}$ avec les centres de $B_{j}$ et $B_{j^{\prime}}$. En répétant le procédé pour chaque arête, le graphe $\Gamma$ se plonge isométriquement dans la réunion des demi-boules $B_{j}$ et des cylindres $C_{i}$. Pour construire le domaine $\Omega_{\varepsilon}$, on va prolonger le cylindre $C_{i}$ en un cylindre $C_{i}^{\prime}$ dont les extrémités seront dans les demi-boules. Chaque boule $B_{j}$ étant vue localement comme un domaine euclidien, on construit une application de $C_{i}^{\prime}=B^{n-1} \times\left[0, l_{i}\right]$ dans la réunion des $C_{i}$ et des voisinages des $B_{j}$ telle que :

- $B^{n-1} \times\left[c \varepsilon, l_{i}-c \varepsilon\right]$ est envoyé isométriquement sur $C_{i}$;

- pour $t \in[0, c \varepsilon]$ et $\left[l_{i}-c \varepsilon, l_{i}\right]$, chaque boule $B^{n-1} \times\{t\}$ est plongée isométriquement dans le voisinage de la demi-boule $B_{j}$ correspondante;

- $B^{n-1} \times\{0\}$ et $B^{n-1} \times\{1\}$ sont envoyé sur le bord équatorial de $B_{j}$;

- l'application obtenue est 1-lipschitzienne.

Les extrémités des cylindres ne sont donc pas isométriques à la métrique produit mais légèrement tordus à l'intérieur des boules :

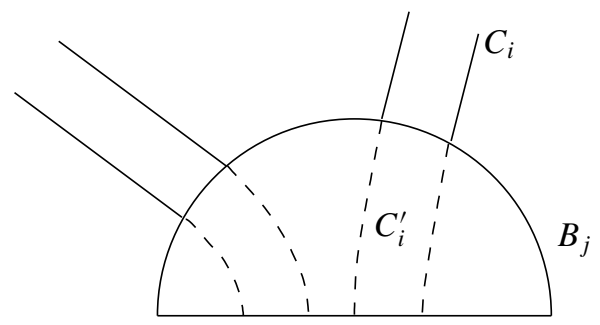

En outre, on fait en sorte que les images de chaque $C_{i}^{\prime}$ soient disjointes (on choisit $c$ assez grand pour que ça soit possible). Ces précisions techniques faciliteront l'étude du spectre. On peut remarquer que quand $\varepsilon$ tend vers $0, \Omega_{\varepsilon}$ tend vers le graphe $\Gamma$ pour la distance de Gromov-Hausdorff. 
En considérant la condition de Steklov sur les boules équatoriales des demi-boules $B_{j}$ et la condition de Neumann sur le reste du bord de $\Omega_{\varepsilon}$, on va montrer que le début du spectre de $\Omega_{\varepsilon}$ tend vers le spectre du graphe, à une constante multiplicative près :

Théorème 4.3. Le $N$-écart spectral entre le spectre de Steklov-Neumann de $\Omega_{\varepsilon}$ et le spectre de $c^{n-1} \Delta_{\Gamma}$, où $\Delta_{\Gamma}$ désigne le laplacien combinatoire sur $\Gamma$, tend vers 0 quand $\varepsilon$ tend vers 0 .

Démonstration. La démonstration se déroule en deux étapes. D'abord, on décompose l'espace des fonctions harmoniques (pour le problème de Steklov-Neumann) sur $\Omega_{\varepsilon}$ en deux sous-espaces pour appliquer le lemme 3.1, puis on montre la convergence en se restreignant à l'un des sous-espaces. On note $\partial \Omega_{\varepsilon, S}$ le bord de Steklov de $\Omega_{\varepsilon}, \partial \Omega_{\varepsilon, S}^{j}, 1 \leq j \leq N$ ses composantes connexes et $\mathscr{H}$ l'espace des fonctions harmoniques sur $\Omega_{\varepsilon}$ vérifiant la condition de Neumann sur $\partial \Omega_{\varepsilon} \backslash \partial \Omega_{\varepsilon, S}$.

Étape 1. On définit l'espace $\mathscr{H}_{0}$ comme étant l'espace des fonctions harmoniques de $\Omega_{\varepsilon}$ constantes sur chacune des $N$ composantes connexes du bord de Steklov. L'orthogonal de $\mathscr{H}_{0}$ pour la forme quadratique $Q$ associée à l'opérateur $\Lambda$ contient les fonction constantes, qui sont aussi dans $\mathscr{H}_{0}$. On définit donc $\mathscr{H}_{\infty}$, comme l'espace des fonctions $Q$-orthogonales à $\mathscr{H}_{0}$ et d'intégrale nulle sur $\partial \Omega_{\varepsilon, S}$. Si $f \in \mathscr{H}_{0}$ et si $g$ est $Q$-orthogonale à $H_{0}$, alors on a, en notant $f_{j}$ la valeur de $f$ sur $\partial \Omega_{\varepsilon, S}^{j}$ :

$$
(f, \Lambda g)=\int_{\partial \Omega_{\varepsilon, S}} f \frac{\partial g}{\partial v}=\sum_{j} f_{j} \int_{\partial \Omega_{\varepsilon, S}^{j}} \frac{\partial g}{\partial v} .
$$

Comme $(f, \Lambda g)$ est nul pour tout $f \in \mathscr{H}_{0}$, on en déduit que $\int_{\partial \Omega_{\varepsilon, S}^{j}} \frac{\partial g}{\partial v}=0$ pour tout $j$. On a donc

$$
\mathscr{H}_{\infty}=\left\{f \in \mathscr{H}: \int_{\partial \Omega_{\varepsilon, S}} f=0, \int_{\partial \Omega_{\varepsilon, S}^{j}} \frac{\partial f}{\partial v}=0 \text { pour tout } j\right\} .
$$

On doit minorer la forme quadratique $Q$ sur l'espace $\mathscr{H}_{\infty}$. Pour cela, on va passer par l'intermédiaire du domaine $D=\bigcup_{j} B_{j}$. Mais comme la restriction des formes harmoniques de $\Omega_{\varepsilon}$ ne vérifient pas la condition de Neumann sur les hémisphères qui bordent les $B_{j}$ on va d'abord reformuler l'expression du bas du spectre de $Q$ sur $\mathscr{H}_{\infty}$. On pose, pour toute fonction $\tilde{f} \in C^{\infty}(F)$ telle que $\int_{\partial \Omega_{\varepsilon, S}} \tilde{f}=0$,

$$
\tilde{Q}(\tilde{f})=\inf \left\{\int_{\Omega_{\varepsilon}}|\mathrm{d} f|^{2}:\left.f\right|_{\partial \Omega_{\varepsilon, S}}=\tilde{f}, \int_{\partial \Omega_{\varepsilon, S}^{j}} \frac{\partial f}{\partial \nu}=0\right\}
$$

On peut vérifier que la borne inférieure de $\tilde{Q}$ (pour $\|\tilde{f}\|_{2}=1$ ) coïncide bien avec le bas du spectre de $Q$ sur $\mathscr{H}_{\infty}$.

On définit les espaces $\mathscr{H}^{D}, \mathscr{H}_{\infty}^{D}$ et les formes quadratiques $Q^{D}, \tilde{Q}^{D}$ en remplaçant $\Omega_{\varepsilon}$ par $D$ dans les définitions de $\mathscr{H}, \mathscr{H}_{\infty}, Q$ et $\tilde{Q}$. Comme $D \subset \Omega_{\varepsilon}, \tilde{Q}$ est minoré par la forme quadratique $\tilde{Q}^{D}$. La première valeur propre de $Q_{\mathscr{H}_{\infty}}$ est donc minorée par la première valeur propre de $Q_{\mathscr{H}_{\infty}}^{D}$. Le domaine $D$ possède $N$ composantes connexes, donc la multiplicité de 0 dans spectre de Steklov de $D$ est $N$, les fonctions propres étant les fonctions constantes sur chaque $B_{j}$. La première valeur propre de $Q_{\mathscr{H}_{\infty}}^{D}$ est donc la $(N+1)$-ième valeur propre de $D$, qui est la première valeur propre non nulle $\sigma_{1}(B(\varepsilon))$ de la demi-boule de rayon $c \varepsilon$. Cette valeur propre se comporte comme $\varepsilon^{-2}$ quand $\varepsilon \rightarrow 0$, ce qui permet d'appliquer que lemme 3.1. 
Étape 2. On doit maintenant comparer les spectres de $Q_{\mathscr{H}_{0}}$ et de la forme quadratique $q$ associée au laplacien combinatoire sur $\Gamma$. Les deux domaines des formes quadratiques sont en bijection de manière évidente, en identifiant une fonction sur les sommets $s_{j}$ de $\Gamma$ avec une fonction constante sur chaque $F_{j}$, prenant les mêmes valeurs. Les normes sur les deux espaces sont différentes. On notera $|\cdot|_{\Gamma}$ la norme euclidienne canonique sur $\mathbb{R}^{S}$, la norme sur $\mathscr{H}_{0}$ est alors $|\cdot|=(c \varepsilon)^{n-1} \omega_{n-1}|\cdot|_{\Gamma}$, où $\omega_{n-1}$ désigne le volume de la boule euclidienne canonique de dimension $n-1$.

Étant donnée $f$ est une fonction sur $S$, on construit une fonction test $\bar{f}$ sur $\Omega_{\varepsilon}$ prenant les mêmes valeurs que $f$ sur chaque $F_{j}$, constante sur chaque demi-boule $B_{j}$ et prolongée de manière affine sur les cylindres $C_{i}$ constituant le domaine $\Omega_{\varepsilon}$. On a

$$
Q(f) \leq Q(\bar{f})=\int_{\Omega_{\varepsilon}}|\mathrm{d} \bar{f}|^{2}=\varepsilon^{n-1} \omega_{n-1} \sum_{a_{i} \in A} \frac{1}{l_{i}-2 c \varepsilon}\left(f\left(x_{i}\right)-f\left(y_{i}\right)\right)^{2},
$$

où $x_{i}$ et $x_{i}$ sont les extrémités de l'arête $a_{i}$, donc $\limsup _{\varepsilon \rightarrow 0} Q(f) /|f|^{2} \leq c^{n-1} q(f) /|f|_{\Gamma}^{2}$.

Réciproquement, étant donné une fonction $f \in \mathscr{H}_{0}$, on construit une fonction test sur les arêtes du graphe $\Gamma$. À partir de la donnée de $f$ sur l'image d'un cylindre $C_{i}^{\prime}=B^{n-1} \times\left[0, \varepsilon, l_{i}\right]$, on définit une fonction $\tilde{f}$ sur l'intervalle $\left[0, l_{i}\right]$ par moyennation sur chaque boule $B^{n-1}$, c'est-à-dire que

$$
\tilde{f}(t)=\frac{1}{\varepsilon^{n-1} \omega_{n-1}} \int_{B^{n-1}} f(x, t) \mathrm{d} x .
$$

On a alors, en utilisant le fait que plongement de $C_{i}^{\prime}$ dans $\Omega_{\varepsilon}$ est 1-lipschitzien,

$$
|\mathrm{d} \tilde{f}|^{2} \leq \frac{1}{\varepsilon^{n-1} \omega_{n-1}} \int_{B^{n-1}}|\mathrm{~d} f|^{2} \mathrm{~d} x
$$

On obtient ainsi une fonction $\tilde{f}$ sur $\Gamma$ qui est $C^{1}$, qui coïncide avec $f$ sur les sommets et qui vérifie $\varepsilon^{n-1} \omega_{n-1}\|\mathrm{~d} \tilde{f}\|^{2} \leq \int_{\Omega_{\varepsilon}}|\mathrm{d} f|^{2}=Q(f)$. Comme sur le graphe, on a $\|\mathrm{d} \tilde{f}\|^{2} \geq q(f)$, on obtient que

$$
\liminf _{\varepsilon \rightarrow 0} \frac{Q(f)}{|f|^{2}} \geq \frac{c^{n-1} q(f)}{|f|_{\Gamma}^{2}}
$$

On a finalement montré que $Q(f) /|f|^{2}$ converge simplement vers $c^{n-1} q(f) /|f|_{\Gamma}^{2}$ quand $\varepsilon \rightarrow 0$. Comme on travaille sur des espaces de dimension finie, cela suffit pour assurer la convergence du spectre et des espaces propres des deux opérateurs.

4C. Application à la prescription de spectre. On a maintenant tous les ingrédients pour montrer le théorème 1.2. La méthode la plus directe serait d'utiliser le théorème 3.12 de convergence du spectre vers celui du bord et les résultats de prescription obtenus dans [Colin de Verdière 1987] (on peut les adapter de manière à prescrire la classe conforme). Cependant, cette méthode ne fonctionne que si la dimension du bord est au moins 3. On va donc procéder autrement en utilisant les plongements de graphes construits au paragraphe précédent. 
Démonstration du théorème 1.2. D'après le théorème 4.2, il existe un graphe complet $\Gamma$ ayant le spectre voulu, avec la propriété de stabilité. On va transplanter ce spectre dans la variété $M$ en commençant par traiter le cas $\rho=1$.

On commence par déformer la variété $M$ en respectant la classe conforme et de manière à pouvoir plonger isométriquement le graphe $\Gamma$ dans $M$ en plaçant les sommets sur $\partial M$. Comme la dimension de $M$ est plus grande que 3, on peut le faire sans que les arêtes se croisent. On note $g$ la métrique obtenue sur $M$.

A priori, la métrique au voisinage du plongement de $\Gamma$ n'est pas euclidienne, on ne peut donc pas plonger isométriquement un ouvert $\Omega_{\varepsilon}$ (construit au paragraphe précédent) au voisinage de $\Gamma$. Cependant, pour tout $\varepsilon$ on peut déformer (de manière non conforme) la métrique $g$ en une métrique $g_{\varepsilon}$ telle que les graphes soient toujours plongés isométriquement et que $\Gamma$ possède un voisinage isométrique au domaine $\Omega_{\varepsilon}$. On peut de plus faire en sorte que $\left(1 / \tau_{\varepsilon}\right) g_{\varepsilon} \leq g \leq \tau_{\varepsilon} g_{\varepsilon}$, pour une famille de réels $\tau_{\varepsilon}>1$ telle que $\tau_{\varepsilon} \rightarrow 1$ quand $\varepsilon \rightarrow 0$.

On peut maintenant appliquer les résultats de convergence spectrale de la section précédente. Pour un $\delta>0$ petit donné, on peut trouver un $\varepsilon$ tel que le $N$-écart spectral entre $\Gamma$ et $\Omega_{\varepsilon}$ soit inférieur à $\delta$. En utilisant les arguments de la démonstration du théorème 3.12 (étape 2), on peut choisir $\varepsilon$ suffisamment petit pour que le $N$-écart spectral entre $\left(\Omega_{\varepsilon}, g_{\varepsilon}\right)$ et $\left(\Omega_{\varepsilon}, g\right)$ soit inférieur à $\delta$. Enfin, on peut faire converger le spectre de $M$ vers celui de $\left(\Omega_{\varepsilon}, g\right)$ d'après le théorème 3.8 , et en particulier déformer $g$ de manière conforme de sorte que le $N$-écart spectral entre $M$ et $\left(\Omega_{\varepsilon}, g\right)$ soit lui aussi inférieur à $\delta$. On peut donc rentre le $N$-écart spectral entre $M$ et $\Gamma$ arbitrairement petit, et ce de manière conforme.

Traitons maintenant le cas où $\rho$ varie. Quitte à multiplier les $a_{i}$ par une constante, on peut supposer que $\rho \leq 1$. Il suffit d'ajouter une étape à la construction précédente et d'utiliser le théorème 3.6 pour faire tendre le spectre de la variété $(M, \rho)$ vers celui de $M$ sans densité.

\section{Multiplicité en dimension 2}

5A. Lignes nodales des fonctions propres. On va montrer dans cette section les obstructions à la prescription de multiplicité en dimension 2 (théorèmes 1.5, 1.7 et 1.10). Comme dans le cas du laplacien, les deux principaux ingrédients sont le théorème nodal de Courant et le théorème de Cheng sur la structure local de l'ensemble nodal. Dans toute la suite du texte, les fonctions propres considérées seront les fonctions harmoniques sur $M$ et pas leur restriction à $\partial M$. En particulier, les lignes et les domaines nodaux seront considérés sur $M$.

Avec ces précisions, le théorème nodal de Courant est valide pour les problèmes de Steklov et SteklovNeumann, quelle que soit la dimension :

Théorème 5.1. Le nombre de domaines nodaux de la k-ième fonction propre du problème de Steklov (ou de Steklov-Neumann) est au plus égal à $k+1$.

La démonstration (essentiellement la même que dans le cas du laplacien) est donnée dans [Kuttler et Sigillito 1969] pour la dimension 2, et elle se généralise immédiatement en toute dimension. 
Contrairement aux fonctions propres du laplacien, les fonctions propres de Steklov ont la particularité que leurs domaines nodaux rencontrent toujours le bord. Cette propriété a déjà été utilisée, par exemple, dans [Bañuelos et al. 2010], et nous y feront appel pour démontrer le théorème 1.7 :

Lemme 5.2. Tout domaine nodal rencontre le bord de la variété. Dans le cas du problème de SteklovNeumann, tout domaine nodal rencontre le bord de Steklov.

Démonstration. Soit $f$ une fonction harmonique non nulle et $D$ un domaine nodal de $f$ ne rencontrant pas le bord de Steklov de la variété. Comme $f$ est harmonique et nulle sur le bord de $D$ (ou vérifie la condition de Neumann le long du bord de Neumann de la variété), elle est uniformément nulle dans $D$. Par conséquent, elle est nulle partout.

S. Y. Cheng [1976] a décrit la structure locale de l'ensemble nodal des fonctions propres du laplacien en dimension 2. On peut les appliquer aux fonctions harmoniques, et le lemme qui précède permet de préciser certaines propriétés topologiques des domaines et des lignes nodales, en particulier leur incompressibilité (une partie d'une surface est dite incompressible si son groupe fondamental s'injecte dans celui de la surface). L'énoncé qui suit rassemble ces résultats :

Théorème 5.3. Supposons que $M$ est de dimension 2 , et soit $f$ une fonction propre du problème de Steklov. Alors :

(1) Les domaines nodaux de $f$ sont incompressibles.

(2) L'ensemble nodal de $f$ intérieur à $M$ est la réunion d'un nombre fini de courbes $C^{2}$ qui sont soit des cercles immergés, soit des arcs immergés dont les extrémités sont sur $\partial M$.

(3) La réunion de ces courbes forme un graphe fini dont les composantes connexes sont incompressibles.

(4) Soit $p$ un point intérieur à M. Si p est un point critique de $f$ situé sur l'ensemble nodal et que l'ordre d'annulation de $f$ en $p$ est $k$, alors au voisinage de p l'ensemble nodal est la réunion de $k$ courbes s'intersectant en p, de courbure géodésique nulle en p et formant un système équiangulaire (en particulier, les sommets du graphe nodal intérieur à $M$ sont de degré pair).

(5) Tout point du bord où $f$ s'annule est l'extrémité d'une ligne nodale intérieure à $M$.

(6) Chaque composante connexe du bord contient un nombre pair d'extrémités du graphe nodal.

(7) Dans le cas du problème de Steklov-Neumann, si p est un point du bord de Neumann où $f$ s'annule, l'ordre d'annulation $k$ de $f$ en $p$ est fini et le point $p$ est un zéro isolé en restriction à $\partial M_{N}$. Au voisinage de $p$ dans $M$, l'ensemble nodal est la réunion de $k$ arcs partant de $p$, de courbure géodésique nulle en $p$ et dont l'extension par réflexion par rapport au bord forme un système équiangulaire.

On appliquera en particulier les propriétés d'incompressibilité au cas du disque. On obtient alors :

Corollaire 5.4. Si M est homéomorphe à un disque, alors les domaines nodaux sont homéomorphes à des disques et les composantes connexes du graphe nodal sont des arbres. 
Démonstration du théorème 5.3. Comme $\Delta f=0$ dans l'intérieur de $M$, on peut appliquer le théorème 2.5 de [Cheng 1976]. En particulier, l'ensemble nodal est la réunion de courbes immergées (les lignes nodales) qui sont localement en nombre fini, cette réunion étant homéomorphe à un graphe localement fini. Cependant, comme l'intérieur de la surface n'est pas compact, on doit vérifier la finitude globale du graphe nodal, qui découle des deux points suivants :

(i) le nombre de lignes nodales est fini;

(ii) les points d'intersection des lignes nodales sont en nombre fini.

Le point (i) se déduit du théorème de Courant : le nombre de domaines délimités par un ensemble de lignes nodales est au moins égal au nombre de ces lignes; par conséquent le nombre total de lignes nodales est majoré par le nombre de domaines nodaux, en particulier il est fini. On montre le point (ii) à l'aide de la formule d'Euler-Poincaré appliquée à la surface : comme les domaines nodaux sont en nombre fini et que leur caractéristique d'Euler est majorée par 1, la caractéristique d'Euler du graphe nodal est minorée en fonction de la topologie de la surface et du nombre de domaines. Or, les sommets du graphe sont de deux types : d'une part les sommets situés sur le bord de la surface aux extrémités des lignes nodales, qui sont en nombre fini et de degré fini car il n'y a qu'un nombre fini de les lignes nodales; d'autre part les intersections de lignes, qui sont de degré au moins 4. Si les sommets intérieurs sont en nombre infini, la caractéristique d'Euler du graphe serait donc $-\infty$, ce qui contredit la formule d'Euler-Poincaré.

On en déduit de ce qui précède les points (2) et (4) du théorème et le fait que le graphe nodal est fini.

Soit $D$ un domaine nodal et $\gamma$ une courbe de $D$ non contractile dans $D$. Si $\gamma$ est contractile dans $M$, alors elle entoure un domaine nodal $D^{\prime}$ distinct de $D$. En outre, $\gamma$ sépare $D^{\prime}$ de $\partial M$, ce qui contredit le lemme 5.2. Par conséquent, $D$ est incompressible. Le même argument montre l'incompressibilité du graphe nodal. On obtient ainsi les points (1) et (3).

Montrons le point (5). Supposons que $p$ est un point du bord qui n'est pas l'extrémité d'une ligne nodale. Le point $p$ n'est donc pas situé à la frontière entre deux domaines nodaux, il est contenu dans un domaine nodal $D$ sur lequel on supposera que $f$ est positive. D'après la propriété d'unique prolongement (théorème 2.7), il n'y a pas de ligne nodale le long du bord, on peut donc trouver un petit voisinage $U$ de $p$ délimité par une courbe de niveau $f(x)=\varepsilon$ avec $\varepsilon>0$ petit. En restriction à $D, f$ est la première fonction propre du problème de Steklov-Dirichlet avec condition de Dirichlet sur les ligne nodales qui bordent $D$ à l'intérieur de $M$. Or, si on définit la fonction test $\tilde{f}$ par $\tilde{f}=\varepsilon$ sur $U$ et $\tilde{f}=f$ sur $D \backslash U$, le quotient de Rayleigh de $\tilde{f}$ est strictement plus petit que celui de $f$, ce qui contredit que $f$ soit la première fonction propre sur $D$.

Le fait qu'un nombre pair de lignes nodales rejoigne chaque composante du bord découle du fait que le signe de la fonction propre change chaque fois qu'on traverse une ligne nodale.

Reste à traiter le cas du problème de Steklov-Neumann. On considère deux copies de la variété $M$ qu'on recolle de manière symétrique le long du bord de Neumann et on note $M^{\prime}$ la surface obtenue. Comme le problème de Steklov est conformément invariant en dimension 2, on peut lisser la métrique le long du recollement de manière conforme et symétrique. Les fonctions propres sur $M$ correspondent alors aux fonctions propres sur $M^{\prime}$ qui sont symétriques. On peut en particulier leur appliquer les résultats 
de Cheng (point (4)). La symétrie de la fonction implique la symétrie des lignes nodales (sur $M^{\prime}$ ) au voisinage du bord de Neumann de $M$. On doit encore montrer que le bord de Neumann ne contient pas de ligne nodale : une fonction harmonique $f$ sur $M^{\prime}$ est la partie réelle d'une fonction holomorphe $g$ (en munissant $M^{\prime}$ de la structure complexe induite par la structure conforme). Si $f$ est une fonction propre symétrique, alors la condition de Neumann et l'équation de Cauchy-Riemann implique que $\operatorname{Im}(g)$ est constante le long du bord de Neumann de $M$. On peut choisir $g$ de sorte que cette constante soit nulle, les zéros de $f$ sur le bord de Neumann sont donc les zéros d'une fonction holomorphe. Par conséquent ils sont isolés.

Démonstration du corollaire 5.4. Si $M$ est un disque, l'incompressibilité des domaines nodaux implique qu'ils sont simplement connexes, donc que ce sont des disques.

Les composantes connexes du graphe nodal sont planaires, et leur incompressibilité signifie qu'ils sont sans cycle. Donc ce sont des arbres.

5B. Bornes sur la multiplicité. On peut maintenant démontrer les théorèmes 1.5, 1.7 et 1.10. En ce qui concerne le théorème 1.5, on reprendra les arguments de [Cheng 1976] et [Besson 1980], qui sont moins précis que ceux de [Nadirashvili 1987] mais plus faciles à adapter au problème de Steklov.

Démonstration du théorème 1.5. Supposons que la surface $M$ soit orientable. On note $E_{k}$ l'espace propre associé à la valeur propre $\sigma_{k}(M)$ et $m_{k}$ sa multiplicité. Selon [Besson 1980], si $m_{k}>4 \gamma+2 k+1$, il existe un point $x$ dans l'intérieur de $M$ et une fonction propre $f \in E_{k}$ telle que l'ordre d'annulation de $f$ en $x$ soit strictement supérieur à $2 \gamma+k$. Localement, il existe donc au moins $4 \gamma+2 k+2$ arcs nodaux partant de $p$.

Si on «ferme » la surface en quotientant chaque composante du bord sur un point, tous les arcs nodaux se referment, et il existe donc au moins $2 \gamma+k+1$ lacets distincts $C^{1}$ par morceaux dans l'ensemble nodal. Or, Cheng [1976, Lemma 3.1] a montré que ces lacets décomposent la surface en au moins $k+2$ composantes connexes. La fonction $f$ possède donc au moins $k+2$ domaines nodaux, ce qui contredit le théorème de Courant.

Comme dans [Besson 1980], le cas des surfaces non orientables se traite par passage à un revêtement à deux feuillets. La surface obtenue en quotientant les bords est de caractéristique d'Euler $p=(1-\chi(M)-l)$. Les arguments des [ibid.] donnent alors la majoration $m_{k} \leq 4 p+4 k+3$.

Démonstration du théorème 1.7. On note $E$ l'espace propre associé à la valeur propre $\sigma_{i}(M, \rho, g)$ pour $i=1$ ou 2 .

Soit $p$ un point intérieur au disque. Si $E$ est de dimension au moins 4, il existe une fonction propre non nulle $f \in E$ telle que $f$ et $\mathrm{d} f$ soient nuls en $p$. Le point $p$ est donc un sommet du graphe nodal de $f$ et il en part au moins quatre arêtes. Comme le graphe nodal est un arbre dont les feuilles sont sur le bord, il délimite au moins quatre domaines nodaux. Il y a donc contradiction avec le théorème de Courant.

Supposons maintenant que $i=1$ et que $E$ soit de dimension 3. Si $p_{0}$ est un point du bord, le sous-espace des fonctions $f \in E$ telles que $f\left(p_{0}\right)=0$ est de dimension au moins 2. Pour tout point $p$ du bord distinct de $p_{0}$, il existe donc une fonction $f_{p}$, qu'on supposera de norme 1 , telle que $f(p)=f\left(p_{0}\right)=0$. Comme 
chacun de ces points est nécessairement l'extrémité d'un ligne nodale et que la fonction $f_{p}$ a exactement deux domaines nodaux, $p_{0}$ et $p$ sont les extrémités de l'unique ligne nodale de $f_{p}$.

Le bord est donc partagé en deux intervalles, $I_{p}^{+}$et $I_{p}^{-}$, d'extrémités $p_{0}$ et $p$, sur lesquels la fonction $f_{p}$ est respectivement positive et négative. En faisant tendre $p$ vers $p_{0}$, on peut faire tendre la longueur de $I_{p}^{-}$ vers 0 . Comme les fonctions $f_{p}$ sont normées et que $E$ est de dimension finie, la famille $f_{p}$ admet une limite $f$ (quitte à extraire une sous-famille). La fonction $f$ est alors positive ou nulle sur la totalité du bord, puisque l'évaluation en un point est une forme linéaire continue sur $E$. Par conséquent, $f$ est de signe constant, ce qui est impossible puisque c'est une fonction propre de la valeur propre $\sigma_{1}$.

Démonstration du théorème 1.10. On note $I$ une composante connexe du bord de Neumann $\partial \mathbb{D}_{N}$ et on choisit $k+1$ points distincts $x_{1}, \ldots, x_{k+1}$ dans $I$. Supposons que la multiplicité de $\sigma_{k}\left(\mathbb{D}, \partial \mathbb{D}_{S}, \rho, g\right)$ soit supérieure ou égale à $k+2$. On peut alors trouver une fonction propre $f$ associée à cette valeur propre qui s'annule en tous les points $x_{i}, i \in[1, k+1]$. En vertu du point 6 du théorème 5.3, chaque $x_{i}$ appartient à une composante connexe du graphe nodal qui joint $x_{i}$ à un point du bord de Steklov, ces composantes étant distinctes. L'ensemble nodal sépare donc $\mathbb{D}$ en au moins $k+2$ composantes connexes, ce qui contredit le théorème de Courant.

\section{Bibliographie}

[Agranovich 2006] M. S. Agranovich, “On a mixed Poincaré-Steklov type spectral problem in a Lipschitz domain”, Russ. J. Math. Phys. 13:3 (2006), 239-244. MR 2007j:35038 Zbl 1162.35351

[Alessandrini et Magnanini 1994] G. Alessandrini et R. Magnanini, "Elliptic equations in divergence form, geometric critical points of solutions, and Stekloff eigenfunctions”, SIAM J. Math. Anal. 25:5 (1994), 1259-1268. MR 95f:35180 Zbl 0809.35070

[Ammann 2003] B. Ammann, "A spin-conformal lower bound of the first positive Dirac eigenvalue", Differential Geom. Appl. 18:1 (2003), 21-32. MR 2004e:58052 Zbl 1030.58020

[Bandle 1980] C. Bandle, Isoperimetric inequalities and applications, Monographs and Studies in Mathematics 7, Pitman, Boston, MA, 1980. MR 81e:35095 Zbl 0436.35063

[Bañuelos et al. 2010] R. Bañuelos, T. Kulczycki, I. Polterovich et B. Siudeja, "Eigenvalue inequalities for mixed Steklov problems", pp. 19-34 dans Operator theory and its applications, édité par M. Levitin et D. Vassiliev, Amer. Math. Soc. Transl. Ser. 2 231, Amer. Math. Soc., Providence, RI, 2010. MR 2012e:35176 Zbl 1217.35127

[Besson 1980] G. Besson, "Sur la multiplicité de la première valeur propre des surfaces riemanniennes", Ann. Inst. Fourier (Grenoble) 30:1 (1980), 109-128. MR 81h:58059 Zbl 0417.30033

[Cheng 1976] S. Y. Cheng, "Eigenfunctions and nodal sets", Comment. Math. Helv. 51:1 (1976), 43-55. MR 53 \#1661 Zbl 0334.35022

[Colbois et al. 2011] B. Colbois, A. El Soufi et A. Girouard, "Isoperimetric control of the Steklov spectrum", J. Funct. Anal. 261:5 (2011), 1384-1399. MR 2012m:35328 Zbl 1235.58020

[Colin de Verdière 1986] Y. Colin de Verdière, "Sur la multiplicité de la première valeur propre non nulle du laplacien", Comment. Math. Helv. 61:2 (1986), 254-270. MR 88b:58140 Zbl 0607.53028

[Colin de Verdière 1987] Y. Colin de Verdière, "Construction de laplaciens dont une partie finie du spectre est donnée", Ann. Sci. École Norm. Sup. (4) 20:4 (1987), 599-615. MR 90d:58156 Zbl 0636.58036

[Colin de Verdière 1988] Y. Colin de Verdière, "Sur une hypothèse de transversalité d'Arnol'd", Comment. Math. Helv. 63:2 (1988), 184-193. MR 90c:58183 Zbl 0672.58046

[Dahl 2005] M. Dahl, "Prescribing eigenvalues of the Dirac operator", Manuscripta Math. 118:2 (2005), 191-199. MR $2006 \mathrm{~h}$. 58037 Zbl 1081.58021 
[El Soufi et Ilias 1986] A. El Soufi et S. Ilias, "Immersions minimales, première valeur propre du laplacien et volume conforme", Math. Ann. 275:2 (1986), 257-267. MR 87j:53088 Zbl 0675.53045

[Fraser et Schoen 2011] A. Fraser et R. Schoen, "The first Steklov eigenvalue, conformal geometry, and minimal surfaces", $A d v$. Math. 226:5 (2011), 4011-4030. MR 2012f:58054 Zbl 1215.53052

[Fraser et Schoen 2012] A. Fraser et R. Schoen, "Eigenvalue bounds and minimal surfaces in the ball", preprint, 2012. arXiv 1209.3789v1

[Hassannezhad 2011] A. Hassannezhad, "Conformal upper bounds for the eigenvalues of the Laplacian and Steklov problem", $J$. Funct. Anal. 261:12 (2011), 3419-3436. MR 2012i:58024 Zbl 1232.58023

[Hoffmann-Ostenhof et al. 1999] T. Hoffmann-Ostenhof, P. W. Michor et N. Nadirashvili, "Bounds on the multiplicity of eigenvalues for fixed membranes", Geom. Funct. Anal. 9:6 (1999), 1169-1188. MR 2001i:35066 Zbl 0949.35102

[Jammes 2007] P. Jammes, "Minoration conforme du spectre du laplacien de Hodge-de Rham", Manuscripta Math. 123:1 (2007), 15-23. MR 2008a:58029 Zbl 1127.35027

[Jammes 2008] P. Jammes, "Prescription du spectre du laplacien de Hodge-de Rham dans une classe conforme", Comment. Math. Helv. 83:3 (2008), 521-537. MR 2009f:58046 Zbl 1155.58305

[Jammes 2009] P. Jammes, "Sur la multiplicité des valeurs propres d'une variété compacte", pp. 1-11 dans Actes du Séminaire de Théorie Spectrale et Géométrie, Sémin. Théor. Spectr. Géom. 26, Institut Fourier, St. Martin-d'Héres, 2009. MR 2011d:58075 Zbl 1235.58022

[Jammes 2011] P. Jammes, "Prescription de la multiplicité des valeurs propres du laplacien de Hodge-de Rham", Comment. Math. Helv. 86:4 (2011), 967-984. MR 2851874 Zbl 1248.58016

[Jammes 2012] P. Jammes, "Sur la multiplicité des valeurs propres du laplacien de Witten", Trans. Amer. Math. Soc. 364:6 (2012), 2825-2845. MR 2888230 Zbl 1242.58015

[Karpukhin et al. 2012] M. Karpukhin, G. Kokarev et I. Polterovich, "Multiplicity bounds for Steklov eigenvalues on Riemannian surfaces", preprint, 2012. arXiv 1209.4869

[Kopachevsky et Krein 2001] N. D. Kopachevsky et S. G. Krein, Operator approach to linear problems of hydrodynamics, I: Self-adjoint problems for an ideal fluid, Operator Theory : Advances and Applications 128, Birkhäuser, Basel, 2001. MR 2003e:76003 Zbl 0979.76002

[Kozlov et al. 2004] V. Kozlov, N. Kuznetsov et O. Motygin, "On the two-dimensional sloshing problem”, Proc. R. Soc. Lond. Ser. A Math. Phys. Eng. Sci. 460:2049 (2004), 2587-2603. MR 2005d:76004 Zbl 1068.35107

[Kuttler et Sigillito 1969] J. R. Kuttler et V. G. Sigillito, "An inequality of a Stekloff eigenvalue by the method of defect", Proc. Amer. Math. Soc. 20 (1969), 357-360. MR 38 \#3633 Zbl 0176.09901

[Nadirashvili 1987] N. S. Nadirashvili, "Multiple eigenvalues of the Laplace operator", Mat. Sb. (N.S.) 133(175):2 (1987), 223-237. In Russian ; translated in Math. USSR, Sb. 61 :1 (1988), 225-238. MR 89a:58113 Zbl 0672.35049

[Stekloff 1899] W. Stekloff, "Sur l'existence des fonctions fondamentales", C. R. Acad. Sci. Paris 128:1 (1899), 808-810. JFM 30.0374 .03

[Stekloff 1902] W. Stekloff, "Sur les problèmes fondamentaux de la physique mathématique (suite et fin)", Ann. Sci. École Norm. Sup. (3) 19 (1902), 455-490. MR 1509018 JFM 33.0800.01

[Taylor 1996a] M. E. Taylor, Partial differential equations, I: Basic theory, Applied Mathematical Sciences 115, Springer, New York, 1996. MR 98b:35002b Zbl 0869.35002

[Taylor 1996b] M. E. Taylor, Partial differential equations, II: Qualitative studies of linear equations, Applied Mathematical Sciences 116, Springer, New York, 1996. MR 98b:35003 Zbl 0869.35003

Received 25 Sep 2012. Revised 20 Oct 2013. Accepted 13 Nov 2013.

PIERRE JAMMES: pjammes@unice.fr

Laboratoire J. A. Dieudonné, UMR no. 7351 CNRS UNS, Université de Nice Sophia Antipolis, 06108 Nice Cedex 02, France et

Département de Mathématiques, Université de Nice Sophia Antipolis, Parc Valrose, 06108 Nice Cedex 02, France 


\title{
Analysis \& PDE
}

\author{
msp.org/apde
}

\section{EDITORS}

EDITOR-IN-CHIEF

\author{
Maciej Zworski \\ zworski@math.berkeley.edu \\ University of California \\ Berkeley, USA
}

BOARD OF EDITORS

Nicolas Burq Université Paris-Sud 11, France

nicolas.burq@math.u-psud.fr

Sun-Yung Alice Chang Princeton University, USA

chang@math.princeton.edu

Michael Christ University of California, Berkeley, USA

mchrist@math.berkeley.edu

Charles Fefferman Princeton University, USA

cf@math.princeton.edu

Ursula Hamenstaedt Universität Bonn, Germany

ursula@math.uni-bonn.de

Vaughan Jones U.C. Berkeley \& Vanderbilt University vaughan.f.jones@vanderbilt.edu

Herbert Koch Universität Bonn, Germany koch@math.uni-bonn.de

Izabella Laba University of British Columbia, Canada ilaba@math.ubc.ca

Gilles Lebeau Université de Nice Sophia Antipolis, France lebeau@unice.fr

László Lempert Purdue University, USA lempert@math.purdue.edu

Richard B. Melrose Massachussets Institute of Technology, USA rbm@math.mit.edu

Frank Merle Université de Cergy-Pontoise, France Frank.Merle@u-cergy.fr

William Minicozzi II Johns Hopkins University, USA minicozz@math.jhu.edu

Werner Müller Universität Bonn, Germany mueller@math.uni-bonn.de
Yuval Peres

Gilles Pisier

Tristan Rivière

Igor Rodnianski

Wilhelm Schlag

Sylvia Serfaty

Yum-Tong Siu

Terence Tao

Michael E. Taylor

Gunther Uhlmann

András Vasy

Dan Virgil Voiculescu

Steven Zelditch
University of California, Berkeley, USA

peres@stat.berkeley.edu

Texas A\&M University, and Paris 6

pisier@math.tamu.edu

ETH, Switzerland

riviere@math.ethz.ch

Princeton University, USA

irod@math.princeton.edu

University of Chicago, USA

schlag@math.uchicago.edu

New York University, USA

serfaty@cims.nyu.edu

Harvard University, USA

siu@math.harvard.edu

University of California, Los Angeles, USA

tao@math.ucla.edu

Univ. of North Carolina, Chapel Hill, USA

met@math.unc.edu

University of Washington, USA

gunther@math.washington.edu

Stanford University, USA

andras@math.stanford.edu

University of California, Berkeley, USA

dvv@math.berkeley.edu

Northwestern University, USA

zelditch@math.northwestern.edu

\section{PRODUCTION}

production@msp.org

Silvio Levy, Scientific Editor

See inside back cover or msp.org/apde for submission instructions.

The subscription price for 2014 is US $\$ 180 /$ year for the electronic version, and $\$ 355 /$ year $(+\$ 50$, if shipping outside the US) for print and electronic. Subscriptions, requests for back issues from the last three years and changes of subscribers address should be sent to MSP.

Analysis \& PDE (ISSN 1948-206X electronic, 2157-5045 printed) at Mathematical Sciences Publishers, 798 Evans Hall \#3840, c/o University of California, Berkeley, CA 94720-3840, is published continuously online. Periodical rate postage paid at Berkeley, CA 94704, and additional mailing offices.

APDE peer review and production are managed by EditFLOW ${ }^{\circledR}$ from Mathematical Sciences Publishers.

PUBLISHED BY

- mathematical sciences publishers

nonprofit scientific publishing

http://msp.org/

(C) 2014 Mathematical Sciences Publishers 


\section{ANALYSIS \& PDE}

\section{Volume $7 \quad$ No. $3 \quad 2014$}

Prescription du spectre de Steklov dans une classe conforme

PIERRE JAMMES

Semilinear geometric optics with boundary amplification

JEAN-Francois COUlOMBEL, OliVier GuÈs and MARK Williams

The 1-harmonic flow with values in a hyperoctant of the $N$-sphere

Lorenzo Giacomelli, Jose M. Mazón and SAlvador Moll

Decomposition rank of $\mathscr{L}$-stable $\mathrm{C}^{*}$-algebras

AARON TIKUISIS and WILHELM WINTER

Scattering for a massless critical nonlinear wave equation in two space dimensions

MARTIN SACK

Large-time blowup for a perturbation of the cubic Szegő equation

HAIYAN XU

A geometric tangential approach to sharp regularity for degenerate evolution equations

EduARdo V. TeIXEIRA and José Miguel UrbanO

The theory of Hahn-meromorphic functions, a holomorphic Fredholm theorem, and its appli- 745 cations

JÖRN MÜLlER and AlEXANDER STROHMAIER 